\title{
State of the Art in Locally Advanced and Recurrent Non-metastatic Prostate Cancer
}

\author{
Hema Vankayala, MD¹ and Ulka Vaishampayan, $\mathrm{MD}^{2}$ \\ 1. Medical Oncologist, John D Dingell VA Medical Center, Wayne State University, Detroit; 2. Professor of Oncology, Karmanos Cancer Institute, \\ Wayne State University, Detroit, US
}

\begin{abstract}
Prostate cancer (PC) is the second leading cause of cancer-related death in men in the US. Biochemical relapse is a significant problem, due to the eventual progression to metastatic disease. It is also an opportunity to explore interventions that may have the potential to prevent or delay the occurrence of metastases. Multiple therapies with diverse mechanisms of action have become available for use in metastatic castrateresistant PC and are currently under active investigation in the non-metastatic disease state. The intent of this article is to discuss currently approved therapy in localized and locally advanced PC and to review the state of the art in clinical management within this disease state. We also discuss the novel agents and targets in development, especially for recurrent, non-metastatic PC.
\end{abstract}

\section{Keywords}

Prostate cancer, PSA relapse, localized prostate cancer, locally advanced prostate cancer, castrate resistance

Disclosure: Hema Vankayala, MD, and Ulka Vaishampayan, MD, have no conflicts of interest to declare. No funding was received in the publication of this article. Received: September 4, 2014 Accepted: October 20, 2014 Citation: Oncology \& Hematology Review, 2014;10(2):123-32 DOI: 10.17925/OHR.2014.10.2.123 Correspondence: Ulka Vaishampayan, MD, Professor of Oncology, Karmanos Cancer institute/Wayne State University, 4100 John R, 4233 HWCRC, Detroit MI 48201, US. E: vaishamu@karmanos.org

Prostate cancer (PC) is the second most common malignancy in American men and the second leading cause of cancer-related death in men in the US. It is estimated that in 2014 nearly 233,000 men will be diagnosed with PC, with 29,480 dying from the disease. 1,2 The majority of them are diagnosed as a result of screening, so symptomatic presentation is unusual. Since the introduction of prostate-specific antigen (PSA) screening in US, more than 1.3 million men have been diagnosed with PC and one million of these have undergone treatment. ${ }^{3}$

Over $95 \%$ of PCs are adenocarcinomas and the median age at diagnosis is 66 years. As per the Surveillance, Epidemiology, and End Results (SEER) 18 database, $80 \%$ have localized disease and only $12 \%$ have regional disease at diagnosis. In the same database, 5-year survival rates vary significantly with stage and have been noted to be $100 \%$ for localized and regional stage disease. The risk factors for PC are increasing age, African American descent, and a family history of PC.

\section{Localized Prostate Cancer}

The current clinical management of localized PC depends on the risk features associated with the cancer and a patient's life expectancy. The cancer risk is dependent on the clinical stage, PSA, highest Gleason Score (GS), and disease burden. The prostate volume also affects the treatment choice in some situations. Treatment options vary from watchful waiting (WW) and active surveillance (AS), to radical prostatectomy (RP), external beam radiation therapy (EBRT), brachytherapy, cryoablation, androgen deprivation therapy (ADT), and high-intensity focused ultrasound.

Treatment recommendations and selection are dependent on disease and patient characteristics, along with patient and physician preferences. Several studies have shown that patients with low-grade, localized PC have a low risk for clinical progression within the first 10 to 15 years of diagnosis, so AS and WW are reasonable options. This strategy is also best suited to men with a shorter life expectancy. ${ }^{4-7}$

First-line ADT is seldom indicated in patients with localized PC. The outcomes of men treated with primary ADT, compared with those who were not, was evaluated in a large, retrospective cohort study. ADT is not associated with increased risk for all-cause mortality, or with reduced PC-specific mortality. However, the risk for PC progression on primary ADT was not studied. The likely explanation for the $40 \%$ decline in PCspecific mortality during this time period is earlier detection and definitive curative intervention. ADT is not curative. This study re-affirms that ADT should be reserved for its established role: as palliation for metastatic $\mathrm{PC}$, in men with node-positive PC after RP, or in combination with RT in intermediate- or high-risk PC.8,9

The treatment options for low-risk and very-low-risk patients include AS, WW, or monotherapies such as interstitial prostate brachytherapy, RT, 
and RP, depending on the life expectancy. The data do not provide clearcut evidence for the superiority of one over another. Hence, a balanced discussion, carefully weighing the risks and benefits, and making a decision after factoring in patient and physician choices, is reasonable.

\section{Radical Prostatectomy in Localized Disease}

The role of RP was evaluated by two randomized clinical trials (RCTS) in the pre-PSA era. In both these studies, RP was compared with observation. The updated results from the Veterans' Administration Cooperative Urological Research Group (VACURG), reporting after 20 years of follow-up, failed to show a difference in mortality. ${ }^{10}$ In contrast to this, the recently reported 23.2 year follow-up results from the Scandinavian Prostate Cancer Group-4 trial (SPCG-4 trial) demonstrated an $11 \%$ absolute reduction in PC-related mortality in the RP arm compared with WW. This benefit is seen mainly in men younger than 65 years of age and in those with intermediate risk cancer. ${ }^{11}$

PC screening underwent a revolutionary change with the advent of PSA testing in addition to digital rectal exams. (DRES) It is noteworthy that only $5 \%$ of SPCG-4 patients were diagnosed with the PSA test as compared with contemporary cases where the majority are diagnosed due to an elevated PSA. Hence the current application of the SPCG-4 results is questionable as $>80 \%$ of them were diagnosed with a DRE. Most of today's newly diagnosed PCs are likely to have lower risk disease than that studied by SPCG-4.

The Prostate Cancer Intervention Versus Observation Trial (PIVOT) was conducted after PSA screening was available in practice and showed no significant reduction in all cause or PC mortality with RP. The pooled data from the intermediate and high-risk groups showed a $10 \%$ absolute reduction in all-cause mortality with $\mathrm{RP}$, but this lost its statistical significance with further analysis. ${ }^{12}$

Several randomized trials studied the role of adding ADT to RP in localized PC and all demonstrated no advantage in overall survival (OS). The exception to this was the use of adjuvant ADT after RP revealed pelvic lymph node involvement with malignancy.

Clinically localized tumors with adverse prognostic features can be completely excised with RP and pelvic LND, leaving patients with a reasonable life expectancy. Studies have reported that patients with adverse prognostic features (T3, GS 8-10, PSA >20) had a better OS survival and lower risk for metastatic progression and PC-specific mortality with RP compared with RT, suggesting RP may be a viable alternative for patients who are not candidates for RT or who prefer surgery. ${ }^{13-15}$

The gold standard for surgery is open retropubic RP (RRP); however, this option is invasive with inherent morbidity associated with it. Therefore, patients and surgeons alike have sought out less-invasive surgical options, one of which is robotic-assisted laparoscopic RP (RALP).

\section{The Robotic-assisted Laproscopic Radical Prostatectomy Approach}

The RALP approach has been rapidly adopted since it was introduced in 2000 and is currently the most common approach to RP, accounting for $53 \%$ of RPs in $2008 .{ }^{16}$
Post-RP, positive surgical margin is an independent predictor of biochemical recurrence and PC-specific mortality. Along with tumor characteristics (GS, pathologic stage, PSA), variations in the experience of the surgeons and technique affect surgical margin status. Hu et al. conducted a retrospective observational study of 5,556 RALP and 7,878 open RP cases from 2004 to 2009 from the SEER-Medicare database. In this propensity-adjusted analysis, RALP was associated with fewer positive surgical margins (13.6\% versus $18.3 \%$; odds ratio [OR]: 0.70; $95 \%$ confidence interval [Cl], 0.66-0.75), largely because of fewer RALP positive margins for intermediate risk (15.0 \% versus $21.0 \%$; OR: 0.66; $95 \% \mathrm{Cl}, 0.59-0.75$ ) and high risk (15.1\% versus $20.6 \%$; OR: 0.70; $95 \% \mathrm{Cl}$, $0.63-0.77)$ disease. RALP was also associated with less use of additional cancer therapy within 2 years of surgery (OR: $0.67 ; 95 \% \mathrm{Cl}, 0.57-0.78$ ). No statistically significant difference in the incidence of surgical margins is noted in low-risk disease, irrespective of surgical approach. Limitations include the retrospective nature of the study and the absence of PSA levels to determine biochemical recurrence. The improved RALP surgical margin status may be attributed to better visualization of the prostate capsule and apex during this approach. ${ }^{17}$

The role of RALP with extended lymph node dissection (LND) is evaluated in high-risk disease patients. This study noted positive nodes in one-third of patients, $35 \%$ had organ-confined disease, and a similar percentage had positive margins. The 3-year biochemical recurrence-free survival varied from $45 \%$ to $86 \%{ }^{18}$

The primary challenge associated with low-risk PC is often an overtreatment of disease, while for high-risk PC it is undertreatment. Many patients with high-risk disease who are likely to benefit from aggressive local therapy with curative intent only receive palliative treatment with ADT. Single modality therapies are less effective in this population.

\section{Role of Andogen Deprivation Therapy with Radiation Therapy in Localized and Locally Advanced Prostate Cancer}

Single modality treatments given to high-risk patients with a curative intent have resulted in poor responses and high clinical and biochemical failures ( $>50 \%$ at 5 years). These poor outcomes are observed irrespective of the primary treatment modality. Given these results, a combined approach was explored. The rationale of the combined approach is that the addition of ADT slows the progression of the tumor by eliminating the hormonal stimulus that drives cancer cell proliferation. In vivo animal models have shown that the combined effect of ADT and RT increases overall cell kill and diminishes growth velocity of the surviving cancer cells.

The necessity of multimodal treatment is re-confirmed in a recent study, where 753 National Comprehensive Cancer Network (NCCN) highrisk localized PC patients were identified from a single- institution RP database. A very-high-risk (VHR) cohort was defined by primary pattern of 5 present on biopsy, or $\geq 5$ cores with GS $8-10$, or multiple NCCN highrisk features. These criteria encompassed $15.1 \%$ of the NCCN high-risk cohort. VHR men were at a higher likelihood of metastasis (hazard ratio [HR] 2.75) and cancer-specific mortality (HR 3.44). Compared with highrisk men, VHR men also had a significantly worse 10-year metastasisfree survival (37\% versus $78 \%$ ) and cancer-specific survival (CSS) (62\% versus $90 \%$ ). Identifying these VHR localized PC patients is important as 
they have poor oncologic outcome and it helps us better select optimal candidates for multimodal treatments or clinical trials. ${ }^{19}$

With the advent of more precise modalities of RT-like 3D-conformal RT and intensity-modulated RT (IMRT), safe dose escalation was achieved with limited local tissue toxicities, along with improved biochemical outcomes. Image-guided RT is important for accurate and precise delivery to avoid normal tissue toxicities. Compared with the seven-field IMRT technique, volumetric-modulated arc therapy (VMAT) delivered lower doses to critical structures such as the penile bulb, bladder, and femoral heads, particularly in high-dose regions, with comparable dose delivery to target volumes..$^{20}$ Dose escalation to 78 Gy has shown to improve freedom from clinical and biochemical failure, especially in patients with PSA >10 $\mathrm{ng} / \mathrm{ml}$ at diagnosis. ${ }^{21}$

The two main studies that evaluated the role of long term ADT along with RT are Radiation Therapy Oncology Group (RTOG) 85-31 and European Organisation for Research and Treatment of Cancer (EORTC) 22863.

The RTOG 85-31 evaluated the role of long-term adjuvant goserelin use after definitive RT to observation, in high-risk disease. The 10-year updated results showed a statistically significant $10 \%$ improvement in survival (49 \% versus $39 \%$ ) and a decrease in incidence of local and distant recurrence, favoring the adjuvant therapy arm. Given the indefinite use of ADT in this study until disease progression, compliance was the main challenge with approximately one-third of patients receiving ADT for $<2$ years, for 2 to 5 years, and for $>5$ years, respectively. ${ }^{22}$

EORTC 22863 evaluated long-term ADT in patients with locally advanced disease (World Health Organization [WHO] grade 3 T1-T2 or T3-T4 NO$\mathrm{N} 1)$. In this study the luteinizing hormone-releasing hormone (LHRH) agonist, goserelin was started on the first day of radiation and continued for 3 years. Ten-year clinical disease-free survival (DFS) was significantly better in the combined treatment group (47.7 \% versus $22.7 \%$ ) compared with the RT-alone group (HR 0.42; $\mathrm{p}<0.0001)]$. A significant improvement in 10-year OS and PC-specific mortality was noted in the combined treatment group. In this study, no significant difference in cardiovascular mortality was noted between treatment groups and in patients with and without cardiovascular problems. To date, the evidence for ADT appears to be more compelling than the evidence for dose escalation of radiation.

Two phase III trials have evaluated the role of ADT with, and without, local RT in patients with locally advanced disease. Both these studies showed an improved OS and reduced PC-specific mortality with the addition of ADT. ${ }^{23,24}$

The duration of ADT has been a recurring question. Can these locally advanced PC patients derive equivalent benefit from a shorter duration of ADT? To elucidate this, RTOG 92-02 compared 4 months of ADT to 28 months in men with locally advanced disease (defined as T2C-T4NO and a PSA level $<150 \mathrm{ng} / \mathrm{ml}$ ). At a median follow-up of 11.3 years, longterm ADT significantly improved local progression, distant metastasis, and biochemical failure rates, but no os benefit was noted. However, patients with a GS of 8-10 demonstrated improved OS with long-term ADT (45.1\% versus $31.9 \% ; p=0.0061){ }^{25}$
EORTC 22961 compared 6 months of ADT to 3 years in locally advanced PC patients. In this study, short-term ADT was also associated with inferior survival. ${ }^{26}$

Based on these data and the meta-analysis of RTOG trials, ${ }^{27}$ the PC patients with high-grade (GS of 8-10) and/or locally advanced disease $(\mathrm{T} 3, \mathrm{~T} 4, \mathrm{~N}+)$ who were treated with $\mathrm{RT}$, derived survival benefit with longterm ADT administration.

Several studies have evaluated the threshold for the duration of neoadjuvant ADT (NADT) prior to RT. RTOG 96-01 compared 6 months to 3 months. The 10-year updated results showed that 6-month ADT decreased distant progression, PC-specific mortality, and all-cause mortality, compared with RT alone. In this study, no increase in treatmentrelated morbidity was noted in the first 5 years after randomization. The 3-month ADT has not demonstrated similar benefit.28 So, neoadjuvant hormone therapy (NHT) given for less than 6 months appears to be suboptimal for survival benefit in patients with intermediate- to high-risk PC. However, in the All Ireland Cooperative Oncology Research Group and Canadian study, no difference in OS and patterns of failure was noted with longer NHT duration. ${ }^{29,30}$

Similarly, in intermediate-risk clinically localized patients, 6 months of ADT along with RT, resulted in a significantly higher survival rate, lower PCspecific mortality, and higher survival free of salvage ADT.31,32

RTOG 9910 evaluated the duration of NADT (28 weeks versus 8 weeks) in intermediate-risk PC. The results reported at the American Society for Radiation Oncology (ASTRO) 5th annual meeting, after a median followup of 9 years, failed to show a significant benefit in DFS and incidence of clinical and BCR at 10 years with longer duration of NADT.

So, given these above data, the current standard of care for high-risk and locally advanced disease is RT, specifically in a 3D-conformal RT (3D-CRT) or IMRT technique to a dose of 75-80 Gy in conjunction with long-term ADT in a neoadjuvant, concurrent, or adjuvant setting for approximately 2 to 3 years.

A retrospective study evaluated long-term follow-up data on high-risk PC patients who received RP, RT + ADT, or RT. It showed similar 10year PC-specific survival rates of $92 \%, 92 \%$, and $88 \%$, respectively. No significant difference in the risks for systemic progression or PC deaths was observed with RT + ADT and RRP. The risk for all-cause mortality, however, was greater with RT plus ADT than after RRP (HR, 1.60; $95 \% \mathrm{Cl}, 1.25-2.05 ; \mathrm{p}=0.0002) .{ }^{33}$ In men with high-risk tumors (GS $\geq 8$ or PSA $>10$ ), both overall and PC-specific mortality were statistically significantly lower in the group that underwent RP than the group that received RT. In men with low-risk tumors (GS $\leq 6$ and PSA $\leq 10$ ), there was no difference in PC-specific mortality and a modest but statistically significant difference in overall mortality. Intermediate risk patients are not included in this analysis. The study results are not conclusive due to differences in patient characteristics with the older age and higher incidence of comorbidities in the radiation therapy group. In addition, patients with higher stage and grade of cancer are likely to receive RT, hence influencing the outcomes. Proper randomized comparison between the two modalities can be used to judge which treatment might be superior. ${ }^{34}$ The Prostate Testing for Cancer and Treatment (ProtecT) 
Table 1: Data from Randomized Controlled Trials of Dose-escalated External Beam Radiotherapy for Prostate Cancer

\begin{tabular}{|c|c|c|c|c|c|c|c|c|}
\hline Trial & $\mathrm{N}$ & NAADT & $\begin{array}{l}\text { Control Dose } \\
\text { Fractions }\end{array}$ & $\begin{array}{l}\text { Escalated } \\
\text { Dose }\end{array}$ & $\begin{array}{l}\text { Survival } \\
\text { Escalated }\end{array}$ & $\begin{array}{l}\text { Survival } \\
\text { Control }\end{array}$ & Escalated & Control \\
\hline MRC RT0135 & 843 & All & 64 Gy/32 & 74 Gy/37 & $71 \%$ at 10 years & $71 \%$ at 10 years & Biochemical PFS 55 \% (50-61) & Biochemical PFS 43 \% (38-48) \\
\hline PROG $9509^{36}$ & 393 & None & $70.2 / 39$ & $79.2 / 44$ & $83.4 \%$ & $78.4 \%$ & Biochemical PFS $16 \%$ & Biochemical PFS 32 \% \\
\hline $\mathrm{NKI}^{37}$ & 664 & $22 \%$ & $68 / 34$ & 78/39 & $75 \%$ at 7 years & $75 \%$ at 7 years & FFF ASTRO $57 \%$ & FFF ASTRO $45 \%$ \\
\hline MDACC $^{21}$ & 301 & None & $70 / 35$ & $78 / 39$ & $79 \%$ at 8 years & $78 \%$ at 8 years & FFF $78 \%$ & FFF $59 \%$ \\
\hline GETUG $06^{38}$ & 306 & None & $70 / 35$ & $80 / 35$ & NS & NS & Biochemical relapse rate $28 \%$ & Biochemical relapse rate $39 \%$ \\
\hline
\end{tabular}

$\overline{A S T R O}=$ American Society for Radiation Oncology; FFF = freedom from failure; NAADT = neoadjuvant androgen deprivation therapy; NS = not significant; $P F S=$ progression-free survival.

Table 2: Trials with Adjuvant Chemotherapy in High-risk Prostate Cancer

\begin{tabular}{|c|c|c|c|c|c|c|}
\hline \multirow[t]{2}{*}{ Trial } & \multicolumn{3}{|c|}{ High-risk Criteria } & \multirow[t]{2}{*}{ Chemo Regimen } & \multirow[t]{2}{*}{ Study Arms } & \multirow[t]{2}{*}{ Chemo Sequence } \\
\hline & Stage & PSA & GS & & & \\
\hline RTOG & Any $T$ & 20-100 & $\geq 7$ & Paclitaxel, estramustine, & $A D T+R T$ versus ADT + RT + TEE & Adjuvant \\
\hline 9902 & $\geq \mathrm{T} 2$ & $<100$ & $8-10$ & etoposide (TEE) & & \\
\hline RTOG & Any $T$ & $\leq 150$ & $\geq 9$ & Docetaxel & $A D T+R T$ versus $A D T+R T+$ docetaxel & Adjuvant \\
\hline \multirow[t]{2}{*}{0521} & $\geq \mathrm{T} 2$ & $<20$ & 8 & & & \\
\hline & Any $T$ & 20-150 & $7-8$ & & & \\
\hline SWOG & pT3b, T4, N1 & & $\geq 8$ & MTX & $R P+A D T$ versus $R P+A D T+M T X$ & Adjuvant \\
\hline \multirow[t]{3}{*}{59921} & & & 7 and positive margin & & & \\
\hline & & Preop $>15$ & $>7$ & & & \\
\hline & & Preop $>10$ & $>6$ & & & \\
\hline
\end{tabular}

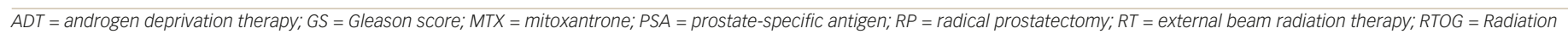
Therapy Oncology Group

trial is currently evaluating prospectively the survival outcomes of AS, RP, and RT in localized PC.

The role of escalated dose RT was evaluated in the randomized trials, ${ }^{35-38}$ as noted in Table 1. These confirmed that it mainly improved biochemical relapse (BCR) rate and biochemical progression-free survival (PFS), but had no significant impact on OS.

\section{Chemotherapy in the Management of High-risk Localized Disease}

Chemotherapy and other systemic agents were used in the adjuvant setting to target the micrometastatic disease and androgen-resistant clones. Several agents were evaluated in combination with ADT and RT in adjuvant and neoadjuvant sequences (see Table 2).

RTOG 99-02 was closed after 4 years, due to excessive thromboembolic events and a significant increase in hematologic and GI toxicity in the adjuvant chemo arm. Long-term complications with myelodysplasia/ acute myelogenous leukemia were noted in three patients. ${ }^{39}$ We await the final results of the RTOG 0521 study, which will certainly help to elucidate the role of chemotherapy in this situation. After a median follow-up of 4.4 years, results from the ADT-alone control arm were reported, showing the estimated 5-year biochemical failure-free survival was $92.5 \%$ (95 \% Cl, 90 to 95), and 5-year OS was $95.9 \%(95 \% \mathrm{Cl}, 93.9$ to 97.9$) .40$

Several new approaches for localized high-risk disease are under evaluation. To name a few: stereotactic Body RT, PI3K and Met inhibitors, and vaccines with, or without, ADT. Along with the additive and synergistic effects of these multimodality therapies on the cancer cells, there is also a potential for additive toxicities.

NCT01546987 is a phase III trial currently recruiting high-risk PC patients to evaluate the addition of steroid 17alpha-monooxygenase TAK-700 to dose-escalated RT and standard ADT.

A Phase III of Cabazitaxel and Pelvic Radiotherapy in Localized Prostate Cancer and High-risk Features of Relapse (PEACE2) is a randomized study currently recruiting patients with high-risk localized PC to evaluate whether the addition of cabazitaxel to standard ADT + RT will improve the clinical PFS. ${ }^{41}$

The treatment of PC does not end with treatment selection; the biochemical response is crucial to evaluate the response, and is delineated below.

\section{The Role of PSA Nadir in Androgen Deprivation Therapy + External Beam Radiation Therapy}

The biochemical response to ADT, and/or RT, is called the 'PSA nadir' (lowest value after treatment) and is useful in predicting outcome and help to guide therapy. Biochemical DFS was significantly higher for patients with a nearly complete biochemical response, as indicated by the pre-RT, post-ADT PSA level.

D'Amico et al. performed a systematic review of two RCTs using the Prentice criteria to assess whether PSA nadir or PSA end concentrations $>0.5 \mathrm{ng} / \mathrm{ml}$ were surrogates for PC-specific mortality. It is noted that men who received a combination of RT and 6 months of ADT were significantly less likely to have both these PSA values $>0.5 \mathrm{ng} / \mathrm{ml}$ compared with 
RT alone $(p<0.0001)$. Both PSA metrics satisfied Prentice criteria for surrogacy. After RT and 6 months of ADT, men with PSA end values $>0.5$ $\mathrm{ng} / \mathrm{ml}$ should be considered for long-term ADT, whereas those with higher-risk disease with a PSA nadir $>0.5 \mathrm{ng} / \mathrm{ml}$ should be considered for inclusion in RCTs investigating drugs that have extended survival in patients with castration-resistant metastatic PC. ${ }^{42}$

RTOG 9413 evaluated prospectively PSA complete response (PSA-CR) (i.e. PSA $\leq 0.3 \mathrm{ng} / \mathrm{ml}$ ) at the end of short-term HT of 4 months as a secondary endpoint. Seventy percent attained PSA-CR. At a median follow-up of 7.2 years, failure to obtain a PSA-CR has a significantly worse disease-specific survival (DSS), DFS, higher incidence of distant metastasis, and biochemical failure. The authors concluded that this will help identify patients who may benefit from the addition of long-term androgen ablation..$^{43}$

One of the main drawbacks of PC screening is overtreatment by at least $30 \%$, as the diagnosis has shifted to low-grade organ-confined disease. It is estimated that $10 \%$ and $45 \%$ of men with low-grade disease were overtreated with radical surgery and RT, respectively. The side effects of local treatments include, but are not limited to, urinary incontinence and impotence, which are significant for individuals who may have been overtreated for their PC. So, the option of targeted focal therapy (TFT) has been explored in this set of population. One of the challenges of TFT is that about two-thirds of these patients have multifocal disease.

\section{The Role of Targeted Focal Therapy in Localized Prostate Cancer}

TFT is defined as the complete ablation of all clinically significant cancer foci within the prostate, using a minimally invasive technique with preservation of the sphincter, normal gland tissue, and the neurovascular bundles. ${ }^{44}$ TFT provides a bridge between surveillance and more definitive therapies.

The Cryosurgery On-Line Data (COLD) registry is the largest data set for patients treated with cryotherapy. A total of 1,160 patients were treated with focal therapy. The focal cryosurgery represented $2.1 \%$ of the treatments in 1999 and had increased to $38.2 \%$ in 2007 in this database. ${ }^{45}$

The cryosurgery series show a biochemical DFS rate of approximately $80 \%$ at 3 to 5 years, with minimal incontinence and high potency rates. The series is faulted for the small number of patients in each series, inconsistent follow-ups, and the use of the ASTRO definition for biochemical recurrence that was intended for use in patients treated with RT. The COLD registry, with large patient numbers, represents a nonstandardized treatment and a compilation of cases from multiple surgeons with variable levels of experience.

The Best Practice Statement on Cryosurgery recommends that patients treated with focal cryotherapy should be included in a registry for future analysis to determine the challenges and safety. Prospective trials with adequate follow-up are necessary and should have precise criteria for patient selection. There is also a need to better define cancer progression. ${ }^{45,46}$ Routine postcryotherapy biopsies are not recommended because of the high negative biopsy rate. However, if PSA is rising, prostate biopsy can be performed, and those patients with positive biopsies be retreated. PSA failures after TFT have the option of additional treatment with cryotherapy, prostatectomy, or RT. ${ }^{44}$ Prostatectomy patients have incontinence rates of $6 \%$ and impotency of $30 \%$ and can take up to 2 years for return of potency after surgery. ${ }^{47}$ Impotence rates after hemigland cryoablation range from 10-30 \% and may take up to 1 year after treatment for return of potency. ${ }^{47}$ Individuals on hormones may experience a longer period before the return of potency. Fistula formation was one of the main concerns with cryotherapy in the 1990s and the incidence in the current literature ranges between $0 \%$ and $0.5 \%$, which is similar to rectal injury after RRP. ${ }^{46}$

Other modalities uses for TFT are high-intensity focal ultrasound (HIFU), where tissue destruction is produced by thermal, mechanical, and cavitation effects with coagulative necrosis. Adequate cell destruction can be produced by short exposure to temperatures of $60^{\circ} \mathrm{C}$ or more. Most of the data is from case series.

Ziglioli et al. identified 13 case series with a total of 5,285 patients, assessing HIFU as a primary or salvage therapy. The vast majority of patients had GS $\leq 7$. Mean pretreatment PSA was $8.54 \mathrm{ng} / \mathrm{ml}$ (standard deviation $[\mathrm{SD}] \pm 1.57 \mathrm{ng} / \mathrm{ml}$ ). The majority used the D'Amico risk classification, and most of them are noted to be low risk. The majority of the case series used Phoenix criteria to define failure and to assess the oncological outcome of the treatment. DFS rate (DFSR) was reported in 10 out of the 13 identified series, DFSR was reported at $\geq 5$ years. When patient stratification in risk groups was reported, the highest DFSR was found in the low-risk group.

The American Urological Association (AUA) and the European Association of Urology (EAU) do not recommend the routine use of HIFU, due to a lack of long-term follow-up data and randomized studies comparing it with conventional therapy options. HIFU is considered a promising minimally invasive treatment for $\mathrm{PC}$, especially in low- and intermediate-risk patients who are not fit for, or are unwilling to undergo, radical surgery. However, as mentioned, long-term follow-up studies are required, in a systematic, randomized, and prospective manner, comparing HIFU to other standard therapeutic strategies. Common agreement on the definitions of failure and positive outcome is of the utmost importance prior to its standardized use. The US Food and Drug Administration (FDA) advisory panel recently denied its use in low-risk PC patients.

The other significant issue is BCR post local therapies. This happens almost a decade after initial PC diagnosis. By this time, the majority of our patients have blossomed into octogenarians and careful treatment considerations seems as crucial as minimizing the treatment toxicities.

\section{PSA Relapse Prostate Cancer Non-metastatic}

Despite successful local therapies, about $40 \%$ of patients eventually experience BCR within 10 years. There is currently no consensus regarding optimal management of this disease state. Options vary depending on the primary treatment received from close surveillance to clinical trial. Precision in defining BCR is important in identifying patients at risk for disease progression. The definition of PSA recurrence is dependent upon the type of local therapy received. The AUA defines post RP PSA recurrence, as the presence of a PSA $>0.2 \mathrm{ng} / \mathrm{ml}$ measured 6-13 weeks after RP, confirmed by a repeat testing with persistence. Ultrasensitive PSA assays have recently improved detection levels to $0.01 \mathrm{ng} / \mathrm{ml}$ and may possibly lead to better treatment outcomes through the earlier adoption of salvage therapies. 
ASTRO defines BCR post-RT, as the midpoint between PSA nadir and the first of three consecutive rises in PSA. An alternative definition of 'nadir + $2 \mathrm{ng} / \mathrm{ml}$ ' (Phoenix definition) has shown improved accuracy over ASTRO in predicting clinical failures.

Not all patients with BCR have the same prognosis, so the stratification of patients into appropriate risk groups is essential. Pre- and posttreatment prognostic factors allow us to assign risk levels and make treatment decisions. The pretreatment factors of prognostic value are absolute baseline PSA, tumor stage (T-stage), and pathologic findings (including GS, surgical margin, and lymph node status). PSA doubling time (PSADT) is the most important prognostic factor for metastasis-free survival and OS. ${ }^{48-51}$

It is important for these patients to undergo complete staging evaluation to rule out metastatic progression prior to subjecting them to any therapy. Conventional imaging with computed tomography (CT) and bone scans is unlikely to reveal metastatic disease at Iow PSA levels. Immunoscintigraphy with Prostascint scanning was used for some time to detect localized recurrence or lymphatic spread. It frequently yielded false negative results and was able to detect about 20-30\% of metastatic disease. The choline positron emission tomography-CT (PET/ $\mathrm{CT}$ ) is an extremely sensitive test, with a low false positivity. It has the capacity to detect disease recurrence in $20 \%$ of patients even with a PSA $\leq 1 \mathrm{ng} / \mathrm{ml} .{ }^{52} \mathrm{As} \mathrm{PC}$ is one of the most common cancers to metastasize to bone, very sensitive bone imaging is needed and the sodium fluoride PET/CT scan is noted to serve this purpose with a high sensitivity (91\%) and specificity (90\%).

BCR is a challenge, as decisions have to be made to delay the onset of metastatic disease and death while avoiding overtreatment, as this disease may never affect patients' os or quality of life.

\section{Management of Post-prostatectomy PSA Relapse}

Recurrence usually manifests initially as PSA rise, with no radiographic evidence of cancer. In the absence of salvage therapy, the median time from PSA recurrence to distant metastasis is about 8 years. The critical concern in these patients is to determine whether a rising PSA reflects local or distant recurrence. Most are candidates for salvage RT and some can benefit from the addition of ADT. Those with unfavorable features benefit from the addition of ADT to RT. Whole pelvic RT confers superior biochemical RFS compared with prostate bed RT for high-risk patients receiving adjuvant or salvage RT along with concurrent androgen suppression. No benefit is noted in low-risk patients. ${ }^{53}$ Stephenson et al. constructed a nomogram to predict the PSA response to salvage RT, in BCR post RP. PSA levels before salvage RT, prostatectomy GS, PSADT, surgical margin status, lymph node involvement, and the use of ADT with salvage RT were identified as significant variables in this model. A better response was noted if salvage RT was initiated before a PSA level of $1 \mathrm{ng} /$ $\mathrm{ml}$. These studies suggest that salvage RT has its best efficacy when initiated early in the course of recurrence.

The best PSA response rates are seen in men with longer PSADTs, but the actual survival benefits are limited to men with short PSADTs. Although a good PSA control is achieved in low-risk men, they are likely to have excellent outcomes regardless of salvage RT. On the contrary, in men with high-risk disease obtaining PSA control is most difficult. These patients actually stand to benefit the most and should not be denied the opportunity for a second chance of cure. Best DFS results are achieved when the salvage RT is given when the PSA is still low $(<0.5)$ and within 2 years of BCR in patients with a PSADT of 6 months. ${ }^{54-56}$ We should not assume that men with a short PSADT have metastatic disease solely based on a short PSADT, and they should be offered aggressive salvage local RT. The recommended radiation dose for salvage $\mathrm{RT}$, is $70 \mathrm{~Gy}$, as noted in the systematic review of 41 studies. It resulted in a higher biochemical PFS compared with a dose of 60 Gy (54 \% versus $34 \%$, respectively). ${ }^{57}$ A $2 \%$ increase in biochemical PFS is noted for each additional Gray of radiation dose.

EORTC 22911 recently reported the long-term results of immediate postoperative RT versus a wait-and-see policy. It included men who were 75 or younger and had undergone RP. They had at least one of the following high-risk features: extracapsular extension, positive surgical margins, or seminal vesicle involvement. About $98 \%$ had no nodal involvement. At a median follow-up of 10.6 years, the biochemical progression rate was $39.4 \%$ in men who received radiation versus $61.8 \%$ in men assigned to the wait-and-see approach $(p<0.0001)$. Locoregional relapse occurred in $8 \%$ of the postoperative RT group versus $17 \%$ in the control group. The benefit noted at 5 years of postoperative RT was not maintained after 10 years, with both groups having similar rates of distant metastasis (about $11 \%$ ) and OS (76.9\% for postoperative irradiation versus $80.7 \%$ for the waitand-see policy). It is noted that younger patients (under age 70) and those with positive surgical margins derive significant benefit from postoperative radiation in terms of biochemical PFS and local control. ${ }^{58}$ Both the EORTC 22911 and SWOG 8794 trials showed a 20-30 \% improvement in biochemical PFS favoring adjuvant RT.

Most of the data about the use of ADT with salvage RT is from retrospective studies. The only randomized study in the salvage setting comes from an abstract presented at the 2011 Genitourinary Cancers Symposium. It consisted of 771 men with a BCR after RP who were randomized to salvage RT and 2 years of bicalutamide (150 mg) versus salvage RT alone in a double-blinded placebo-controlled trial. At a median follow-up of 7 years, there was no survival difference but a higher PSA progressionfree rate $(57 \%$ versus $40 \% ; p<0.0001)$. Lower cumulative incidence of metastatic PC (7 \% versus $13 \%$; $p<0.04$ ) was also noted in the arm on concurrent ADT, with an increased benefit in high-risk disease. ${ }^{59}$

What is the impact of these published RCT on referral patterns? This was evaluated in patients with high-risk features. It is noted that only 13-14 \% of these high-risk patients were referred for adjuvant RT within 6 months of RP and $70 \%$ of these patients had a PSA level of $<0.2 \mathrm{ng} / \mathrm{ml}$ at the time of the referral. It is also noted that patients with higher pathologic T stage and more advanced GS are more likely to be referred. ${ }^{60,61}$ These poor referral patterns can be explained by the ongoing debate regarding the optimal timing of postsurgical RT and by the introduction of ultrasensitive PSA tests capable of detecting levels of $<0.01 \mathrm{ng} / \mathrm{ml}$. It remains controversial as to whether RT delivered at the earliest signs of PSA recurrence is as effective as treatment with an undetectable PSA.

Two phase III RCTS (RADICALS and RAVES) are currently being conducted to compare adjuvant versus early salvage RT. However, until the results of these trials are available, decisions for these patients should be made in 
a multidisciplinary fashion by both urologists and radiation oncologists to discuss the potential benefits and risks for adjuvant RT.

Natural compounds like sulforaphane (SF), which is present in cruciferous vegetables, is evaluated in post-RP PSA relapse PC patients \pm adjuvant or salvage RT. This is a multicenter, double-blind, randomized, placebocontrolled study. A 78 \% increase in PSADT was observed in the SF group (21.9 versus 12.1 months) compared with placebo. The mean change in PSA levels between month 6 and 0 was significantly lower in the SF group compared with placebo $(0.099 \pm 0.341$ versus $0.620 \pm 1.417 \mathrm{ng} / \mathrm{ml}$; $\mathrm{p}=0.03$ ). The medication was pretty well tolerated, other than some $\mathrm{Gl}$ effects. The results were encouraging both in efficacy and safety, indicating the need for more extensive evaluation of this compound. ${ }^{62}$

\section{Salvage Therapy Post-radiation Therapy PSA Relapse}

Various options exist for salvage local treatment after the failure of RT, including surgery, additional RT, and cryotherapy.

Salvage RP after RT has the longest history and best likelihood of local control relative to other salvage treatments. ${ }^{63}$ However, the risks for adverse events (AEs) are high with salvage RP because of the radiation-induced fibrosis and poor wound healing. The best contemporary evidence is from a retrospective, international, multi-institutional cohort analysis of 404 men with radiation-recurrent PC. Five-year BCR-free, metastasis-free, and CSS rates of $48 \%, 83 \%$, and $92 \%$, were noted, respectively, with post salvage RP. Men with a GS $<7$ and preoperative PSA $<4 \mathrm{ng} / \mathrm{ml}$ were identified as a favorable risk. Among this group of favorable patients $(n=120)$, no PC-specific deaths were noted and BCR-free survival was $64 \%$ at 5 years. ${ }^{64}$

In this era of robotic surgery, the role of salvage robotic RP was also evaluated in post-RT BCR. The oncologic outcomes correlated strongly with positive margin rates. About $23 \%$ had positive margins and $30 \%$ had BCR. Although the results appear comparable with open surgery, the small sample size and short follow-up of the study limits data on meaningful endpoints. ${ }^{65}$ Compared with primary RP, salvage $\mathrm{RP}$ is associated with increased risk for bladder neck contractures (6\% versus $47 \%$ ), urinary retention (3.5\% versus $25.3 \%$ ), urinary fistula $(0.06 \%$ versus $4.1 \%)$, abscess $(0.7 \%$ versus $3.2 \%)$, and rectal injury $(0.6 \%$ versus $9 \%) .{ }^{66} \mathrm{~A}$ decline in rectal injury was noted since 2000 . Despite improvements in technology and technique, rates of impotence, urinary incontinence, and bladder neck contracture are still high, suggesting that salvage RP should be performed primarily at high-volume centers by experienced surgeons. ${ }^{67}$

Most of the studies on salvage brachytherapy are small, single-institutional and retrospective in nature, with shorter follow-up, so limited conclusions can be made. In a systematic review of 18 studies, marked variations were noted among the studies, treatment methods, and outcomes. The biochemical DFS at 4 to 5 years ranged from $25 \%$ to $75 \%$, while OS and DSS ranged from $54 \%$ to $94 \%$ and $74 \%$ to $100 \%$, respectively. The crude rate of grade 3-4 genitourinary toxicity was $13 \%(0-47)$ and GI toxicity was $5 \%(0-20) .{ }^{8}$ Higher toxicity rates might impair the widespread use of this approach.

The situation is similar with salvage cryoablation, as most studies are small retrospective series. An analysis of the COLD Registry found a biochemical
DFS at 3 years of $66.7 \% .{ }^{69}$ In this study, a pre-RT PSA $<10 \mathrm{ng} / \mathrm{ml}, \mathrm{GS}<8$, clinical stage T1c or T2 disease, a low presalvage PSA ( $<5 \mathrm{ng} \mathrm{ml}$ ), and a long PSADT (>16 months) are associated with a favorable response. This is similar to the results of the salvage RT studies. ${ }^{70}$ This is an attractive alternative, but randomized trials are needed to elucidate the relative cancer control and toxicity of various options for local salvage after definitive RT.

\section{Hormonal Therapy For PSA-recurrent Prostate Cancer}

The standard of care for BCR PC after maximal local therapy is androgen deprivation, mainly through chemical castration. Gonadotrophin-releasing hormone (GnRH) agonists, including leuprolide and goserelin, have been the primary medical castration therapies, while the GnRH antagonist, degarelix, has recently gained some momentum. Currently, no consensus exists on the ideal timing or PSA cut-point for the institution of therapy. The two main concerns are the timing of ADT (early versus deferred) and continuous versus intermittent therapy.

Until we have the results of the prospective studies, TOAD and ELLAT, uncertainty exists about the OS benefits of immediate versus delayed ADT. As ADT is accompanied by serious AEs and quality-of-life factors, many patients like to defer ADT initiation. Retrospective studies reported median metastasis-free survival of about 10 years in men with BCR post RP, even in the absence of ADT and salvage RT. These data support the earlier findings that patients with long PSADT often enjoy a prolonged PFS. Given the lack of a clear OS advantage with the use of immediate ADT, it is recommended to defer ADT in patients at low risk for metastatic progression (PSADT >9 months; absolute PSA $<10 \mathrm{ng} / \mathrm{ml}$ ), while early initiation remains a reasonable choice for those at high risk for developing metastatic disease (PSADT <6 months; absolute PSA >20 ng/ml).48,71,72

Recently, this question was revisited in the American Society of Clinical Oncology (ASCO) 2014 meeting, when prospective data were presented from the UCSF Cancer of the Prostate Strategic Urologic Research Endeavor


or RT with curative intent were studied. The median age was 69 years, $33.8 \%$ had a GS $>7$, and a third received RT as primary treatment. The median time from primary treatment to PSA relapse was 27 months. Patients were assigned to the 'immediate' strategy if ADT was initiated within 3 months of PSA relapse, and to the 'deferred' strategy if they initiated 2 or more years after PSA relapse or when they presented with metastasis, symptoms, or a short PSADT. The estimated 5-year OS was not significantly different in the immediate and delayed groups (87.2\% versus $85.1 \%)$, and, similarly, the estimated 10-year OS was the same in the two groups (71.6\% versus $71.6 \%$ ). These results reassure us that there is no rush for ADT. ${ }^{73}$

The second controversial decision for BCR PC patients is whether to use intermittent (IAD) or continuous (CAD) ADT. An international randomized phase III trial evaluated this in 1,386 men with BCR following RT (with or without prior prostatectomy). The main idea is to evaluate the noninferiority of IAD to CAD. The IAD group received 8 months of hormonal therapy, followed by treatment withdrawal until PSA reached $10 \mathrm{ng} / \mathrm{ml}$ or higher during the off-treatment period. After a median follow-up of 6.9 years, the OS was noninferior for IAD compared with CAD (8.8 years versus 9.1 years, $\mathrm{HR}, 1.02 ; 95 \% \mathrm{Cl}, 0.86-1.21)$. PC-related deaths were greater in the IAD group (122 versus 97 deaths), while nonprostate deaths were 
lower in the IAD group (134 versus 146 deaths). Men reporting reduced hot flashes were mainly in the IAD arm, but no other differences in AES were reported. IAD is a reasonable standard of care for the management of patients with BCR PC. ${ }^{74}$ The risks and benefits must be weighed for each patient, depending on their concomitant comorbidities and risk factors for the metabolic syndrome.

\section{Other Systemic Therapy Approaches for PSA-recurrent Prostate Cancer}

Several phase I/II studies are available that evaluate the role of hormonal and nonhormonal natural and pharmaceutical agents in this situation. Some of the trials evaluate the combination of ADT and experimental agents. To name a few, the agents used in combination with ADT are sipuleucel-T, bevacizumab, thalidomide, AKT inhibitors, and CTLA4 antibodies. With oral thalidomide, a trend toward increased PSADT is noted in comparison to placebo. ${ }^{75}$ The role of cancer immunotherapy was evaluated in a phase I/II study, where patients with PSA relapse were subjected to dendritic cell vaccine DCVAC/PCa. Patients received a total of 12 doses of subcutaneous injections. Twenty-one patients were evaluated and the median PSADT increased from 7.86 months prior to the treatment, to 26.08 months. Stable PSA responses of about 3 years were noted in eight patients. These results are promising and support the early use of immunotherapy in BCR. ${ }^{76}$

Several nonhormonal agents are being studied in this situation, including celecoxib, a combination of atorvastatin and celecoxib, and disulfiram. Targeted agents such imatinib (Gleevec ${ }^{\circledR}$ ), lapatinib (Tyverb ${ }^{\circledR}$ ), and lenalidomide (Revlimid $^{\circledR}$ ), as well as vaccines such as prostatic acid phosphatase vaccine, are under study. The celecoxib-versus-placebo study was terminated due to cardiovascular concerns, despite significant improvements in PSA velocity. In the calcifidol study, $80 \%$ had increases in PSADT. Lapatinib caused significant reduction in the mean PSA slope, but no PSA responses. ${ }^{77}$ Natural nonhormonal products such as pomegranate (PoMX), curcumin, Acai juice, and indole-3-carbinol are also under investigation.

POMX is a rich source of polyphenols and is reported to have higher antioxidant potential than green tea or red wine. Bioactive compounds in PoMX juice have been shown to have dose-dependent anti-tumor activity in both in vitro cell culture and in vivo mouse model. ${ }^{78}$ Research by Pantuck et al. documented a significant increase in PSADT in PC patients following RP or RT for organ-confined disease. ${ }^{79}$ Paller's study was a randomized, multicenter, double-blind phase II, dose-exploring trial, subjecting men with a rising PSA and without metastases to either $1 \mathrm{~g}$ or $3 \mathrm{~g}$ of POMx. PSADT lengthened in both groups by $>6$ months. Forty-two percent of patients discontinued treatment prior to the protocol-definition of PSA progression, or at 18 months, primarily due to a rising PSA. No significant changes occurred in testosterone levels. The inclusion of a placebo control arm is needed to further investigate the clinical utility of this intriguing supplement.

Muscadine grape skin (MPX) comprising ellagic acid, quercetin, and resveratrol, demonstrates preclinical activity against PC cells in vitro. Phase I data suggested that 4,000 mg of MPX is safe and it extended the PSADT by 3.9 months. ${ }^{80}$ The phase 11 study is currently accruing patients.

Curcumin inhibits nuclear factor kappa-light-chain-enhancer of activated $\mathrm{B}$ cells (NF-kB) and slows PC growth in cell lines. The androgen sensitive and insensitive cell lines were exposed to curcumin and it is noted to be a potent inhibitor of epidermal growth factor-receptor (EGF-R) signaling. ${ }^{81}$ This is accomplished by downregulation of the EGF-R protein, inhibition of the intrinsic and ligand-induced EGFR-tyrosine kinase activity.

The UK National Cancer Research Network (NCRN) Pomi-T study is a double-blind, placebo-controlled randomized trial, evaluated the effect of a polyphenol-rich whole food supplement on PSA progression in men with PC.82 Of 199 men with localized PC, $60 \%$ managed with primary AS or $40 \%$ with WW following prior intervention. They were randomized in 2:1 to receive an oral capsule containing a blend of PoMX, green tea, broccoli and turmeric, or an identical placebo for 6 months. The median rise in PSA was $14.7 \%$ versus $78.5 \%$ with placebo $(p=0.0008)$. Forty-six percent of men in the food supplement arm had stable or lower PSA at trial completion versus $14 \%$ in the placebo arm. The supplement was well tolerated except for mild GI symptoms. This study showed short-term, favorable effect on PSA kinetics with this food supplement, but randomized trials with longterm follow-up are required to confirm this effect.

\section{Non-metastatic Castrate-resistant Prostate Cancer}

The non-metastatic castrate-resistant PC (NM-CRPC) develops mainly in the setting of the early use of ADT. NM-CRPC has a heterogeneous natural history, but can be enriched based on known predictors of PC-related morbidity and/or mortality.

ARN-509 is a novel second-generation anti-androgen that binds directly to the ligand-binding domain of the androgen receptor, impairing nuclear translocation, and DNA binding. The phase II portion of a multicenter phase I/II study evaluated the activity in high-risk NM-CRPC patients. All patients had CRPC, no radiographic evidence of metastases (pelvic lymph nodes $<3 \mathrm{~cm}$ below the iliac bifurcation were allowed), and high risk for disease progression based on PSA value $\geq 8 \mathrm{ng} / \mathrm{ml}$ within 3 months of enrollment and/or PSADT $\leq 10$ months. Patients received ARN-509 at $240 \mathrm{mg} /$ day. The primary endpoint was PSA response rate at 12 weeks according to the Prostate Cancer Working Group 2 (PCWG2) criteria. A total of 47 patients were enrolled, $30 \%$ with GS 8-10 and median PSA of $10.7 \mathrm{ng} / \mathrm{ml}$. All patients received prior treatment with a LHRH analog, with or without a first-generation anti-androgen. The 12week PSA response was $91 \%$ and the time to PSA progression has not been reached. The most common treatment-related AEs were fatigue (30\%), diarrhea (28\%), nausea (17\%), rash (13\%), and abdominal pain (11\%). The incidence of grade 3 AEs was $6.4 \%$, and no seizures have been observed to date. ${ }^{83}$

A similar study design with ODM-201, an androgen receptor inhibitor from Orion Pharmaceuticals, is ongoing. In addition, a global phase III clinical trial, known as PROSPER, will evaluate the safety and efficacy of enzalutamide in patients with NM-CRPC.

MVA-BN ${ }^{\circledR}-\mathrm{PRO}$ is an investigational PC immunotherapy comprising a highly attenuated nonreplicating vaccinia virus, engineered to encode PSA and prostate acid phosphatase (PAP) proteins. In a trial, patients included those with PSA relapse PC while on androgen supression therapy (AST) who were chemotherapy naïve. The primary endpoint was safety. Immune responses (humoral and cellular) to MVA-BN ${ }^{\circledR}-\mathrm{PRO}$, 
including the transgenes PSA and PAP, were assessed by the enzymelinked immunosorbent assay (ELISA) and enzyme-linked immunospot (ELISPOT) assays, respectively. Twenty-four subjects were dosed. All subjects completed the initial three vaccinations (treatment) and 21 subjects received six vaccinations (re-treatment). Seven responders received additional vaccinations during the extended treatment. No doselimiting toxicity (DLT) were reported. There were no reported $\geq$ grade 3 treatment-related AE. The most common related AEs were grade 1 or 2 general disorders and administration site reactions. ${ }^{84}$

The IMAAGEN core study is a phase II, multicenter study evaluating the ability of abiraterone plus prednisone to decrease PSA levels in patients with NM-CRPC with rising PSA. They have enrolled 131 patients with PSA value $\geq 10 \mathrm{ng} / \mathrm{ml}$ or PSADT $\leq 10$ months at screening and 122 are evaluable. Median age was 72 years (range 48-90) and median baseline PSA was $13.7 \mathrm{ng} / \mathrm{ml}(1.6-167.8 \mathrm{ng} / \mathrm{ml})$. By the end of six cycles of treatment,
$60 \%(73 / 122)$ had $\geq 90 \%$ PSA reduction. From these results the authors concluded that the rate and depth of PSA decline appeared superior than in men with metastatic CRPC (MCRPC). ${ }^{85}$

\section{Conclusion}

$\mathrm{PC}$ is heterogeneous with a variable disease course. Careful consideration needs to be made in treatment selections for our aging population. The real problem is BCR. There is no consensus regarding optimal management of this disease state. BCR should be individualized, taking into account risk stratification and prior therapy, before making a treatment decision. The benefit of the early initiation of ADT is offset by its AEs. Patients placed on surveillance face anxiety and seek interventions to delay or prevent progression to metastatic disease. However, PSA relapse represents a window of opportunity to evaluate therapies that will delay progression. Given that the majority of these patients have been pretreated with ADT, NM-CRPC is on the rise. Clinical trials should be strongly considered in the setting of BCR, especially for NM-CRPC.
1. Rullis I, Shaeffer JA, Lilien OM, Incidence of prostatic carcinoma in the elderly, Urology, 1975;6:295-7.

2. Sakr WA, et al., High grade prostatic intraepithelial neoplasia (HGPIN) and prostatic adenocarcinoma between the ages of 20-69: an autopsy study of 249 cases, In Vivo, 1994;8:439-43.

3. Welch $\mathrm{HG}$, Albertsen PC, Prostate cancer diagnosis and treatment after the introduction of prostate-specific antigen screening: 1986-2005, J Natl Cancer Inst, 2009;101:325-9.

4. Klotz L, Active surveillance with selective delayed intervention for favorable risk prostate cancer, Urol Oncol, 2006;4:46-50.

5. Johansson JE, et al., Natural history of early, localized prostate cancer, JAMA, 2004:291:2713-9.

6. Zietman AL, et al., Conservative management of prostate cance in the prostate specific antigen era: the incidence and time course of subsequent therapy, J Urol, 2001;166:1702-6.

7. Adolfsson J, et al., Localized prostate cancer and 30 years of follow-up in a population-based setting, Prostate Cancer Prostatic Dis, 2000;3:37-42.

8. Potosky AL, et al., Effectiveness of primary androgendeprivation therapy for clinically localized prostate cancer, I Clin Oncol, 2014:32:1324-30.

9. Nelson JB, Observation for Clinically Localized Prostate Cancer, J Clin Oncol, 2014;32:1295-8

10. Iversen P, Madsen PO, Corle DK, Radical prostatectomy versus expectant treatment for early carcinoma of the prostate. Twenty-three year follow-up of a prospective randomized study, Scand I Urol Nephrol Suppl, 1995;172:65-72.

11. Bill-Axelson A, et al., Radical prostatectomy or watchful waiting in early prostate cancer, N Eng/ J Med, 2014;370:932-42.

12. Wilt TJ, et al., Radical prostatectomy versus observation for localized prostate cancer, N Eng/ J Med, 2012;367:203-13.

13. Lau WK, et al., Radical prostatectomy for pathological Gleason 8 or greater prostate cancer: influence of concomitant pathological variables, J Urol, 2002;167:117-22.

14. Zelefsky MJ, et al., Metastasis after radical prostatectomy or external beam radiotherapy for patients with clinically localized prostate cancer: a comparison of clinical cohorts adjusted for case mix, I Clin Oncol, 2010:28:1508-13.

15. Abdollah F, et al., A competing-risks analysis of survival after alternative treatment modalities for prostate cancer patients: 1988-2006, Eur Urol, 2011;59:88-95.

16. Hu JC, et al., Comparative effectiveness of minimally invasive vs open radical prostatectomy, JAMA, 2009;302:1557-64.

17. Hu IC, et al., Comparative effectiveness of robot-assisted versus open radical prostatectomy cancer control, Eur Urol, 2014:66:666-72.

18. Yuh B, et al., The role of robot-assisted radical prostatectomy and pelvic lymph node dissection in the management of high-risk prostate cancer: a systematic review, Eur Urol, 2014;65:918-927.

19. Sundi $D$, et al., Very-high-risk localized prostate cancer: definition and outcomes, Prostate Cancer Prostatic Dis, 2014;17:57-63.

20. Davidson MT, et al., Assessing the role of volumetric modulated arc therapy (VMAT) relative to IMRT and helical tomotherapy in the management of localized, locally advanced, and post-operative prostate cancer, Int J Radiat Oncol Biol Phys, 2011;80:1550-8

21. Kuban DA, et al., Long-term results of the M. D. Anderson randomized dose-escalation trial for prostate cancer, Int J Radiat Oncol Biol Phys, 2008:70:67-74.

22. Pilepich MV, et al., Androgen suppression adjuvant to definitive radiotherapy in prostate carcinoma - long-term results of phase III RTOG 85-31, Int J Radiat Oncol Biol Phys, 2005:61:1285-90.

23. Widmark $A$, et al., Endocrine treatment, with or without radiotherapy, in locally advanced prostate cancer (SPCG-7/SFUO 3): an open randomised phase III trial, Lancet, 2009;373:301-8.
24. Warde P, et al., Combined androgen deprivation therapy and radiation therapy for locally advanced prostate cancer: a randomised, phase III trial, Lancet, 2011:378:2104-11.

25. Horwitz EM, et al., Ten-year follow-up of radiation therapy oncology group protocol 92-02: a phase III trial of the duration of elective androgen deprivation in locally advanced prostate cancer, J Clin Oncol, 2008;26:2497-504.

26. Bolla $\mathrm{M}$, et al., Duration of androgen suppression in the treatment of prostate cancer, N Engl J Med, 2009;360:2516-27.

27. Roach $\mathrm{M}, 3$ rd, et al., Predicting long-term survival, and the need for hormonal therapy: a meta-analysis of RTOG prostate cance trials, Int I Radiat Oncol Biol Phys, 2000:47:617-27.

28. Denham J.W, et al., Short-term neoadjuvant androgen deprivation and radiotherapy for locally advanced prostate cancer: 10-year data from the TROG 96.01 randomised trial, Lancet Oncol, 2011;12:451-9.

29. Armstrong JG, et al., A randomized trial (Irish clinical oncology research group 97-01) comparing short versus protracted neoadjuvant hormonal therapy before radiotherapy for localized prostate cancer, Int I Radiat Oncol Biol Phys, 2011;81:35-45

30. Crook J, et al., Final report of multicenter Canadian phase III randomized trial of three versus eight months of neoadjuvan androgen deprivation therapy before conventional-dose radiotherapy for clinically localized prostate cancer, Int J Radiat Oncol Biol Phys, 2009;73:327-33.

31. D'Amico AV, et al., Six-month androgen suppression plus radiation therapy vs radiation therapy alone for patients with clinically localized prostate cancer: a randomized controlled trial, JAMA, 2004:292:821-7.

32. D'Amico AV, et al., Duration of short-course androgen suppression therapy and the risk of death as a result of prostate cancer, I Clin Oncol, 2011;29:4682-7.

33. Boorjian SA, et al., Long-term survival after radical prostatectomy versus external-beam radiotherapy for patients with high-risk prostate cancer, Cancer, 2011:117:2883-91.

34. Hoffman RM, et al., Mortality after radical prostatectomy or external beam radiotherapy for localized prostate cancer, I Natl Cancer inst, 2013:105:711-8.

35. Dearnaley DP, et al., Escalated-dose versus control-dose conformal radiotherapy for prostate cancer: long-term results from the MRC RT01 randomised controlled trial, Lancet Oncol, 2014;15:464-73.

36. Zietman $\mathrm{AL}$, et al., Randomized trial comparing conventionaldose with high-dose conformal radiation therapy in early-stage adenocarcinoma of the prostate: Iong-term results from proton radiation oncology group/American College of Radiology 95-09, J Clin Oncol, 2010;28:1106-11.

37. Al-Mamgani A, et al., Update of Dutch multicenter doseescalation trial of radiotherapy for localized prostate cancer, Int J Radiat Oncol Biol Phys, 2008:72:980-8.

38. Beckendorf $\mathrm{V}$, et al., 70 Gy versus $80 \mathrm{~Gy}$ in localized prostate cancer: five-year results of GETUG 06 randomized trial, Int । Radiat Oncol Biol Phys, 2011:80:1056-63.

39 Rosenthal SA et al, Phase III multi-institutional trial of adjuvant chemotherapy with paclitaxel, estramustine, and oral etoposide combined with long-term androgen suppression therapy an radiotherapy versus long-term androgen suppression plus radiotherapy alone for high-risk prostate cancer: preliminary toxicity analysis of RTOG 99-02, Int J Radiat Oncol Biol Phys, 2009:73:672-8.

40. Dorff TB, et al., Adjuvant androgen deprivation for high-risk prostate cancer after radical prostatectomy: SWOG $\$ 9921$ study J Clin Oncol, 2011;29:2040-5

41. Fizazi K, et al., A randomized phase III, factorial design, of cabazitaxel and pelvic radiotherapy in patients with localized prostate cancer and high-risk features of relapse, ASCO Meeting Abstracts, 2014;32(Suppl. 15):TPS5098.

42. D'Amico AV, et al., Surrogate endpoints for prostate cancerspecific mortality after radiotherapy and androgen suppression therapy in men with localised or locally advanced prostate cancer: an analysis of two randomised trials, Lancet Oncol, 2012:13;189-95.

43. Cury FL, et al., Prostate-specific antigen response after shortterm hormone therapy plus external-beam radiotherapy and outcome in patients treated on Radiation Therapy Oncology Group study 9413, Cancer, 2013:119:1999-2004.

44. Crawford ED, Barqawi A, Targeted focal therapy: a minimally invasive ablation technique for early prostate cancer, Oncology (Williston Park), 2007;21:27-32; discussion 33-4, 39.

45. Ward JF, Jones JS, Focal cryotherapy for localized prostate cancer: a report from the national Cryo On-Line Database (COLD) Registry, BJU Int, 2012;109:1648-54.

46. Babaian RJ, et al., Best practice statement on cryosurgery for the treatment of localized prostate cancer, J Urol, 2008:180:1993-2004.

47. Onik G, et al., The "male lumpectomy": focal therapy for prostate cancer using cryoablation results in 48 patients with at least two-year follow-up, Urol Oncol, 2008;26:500-5.

48. Antonarakis ES, et al., The natural history of metastatic progression in men with prostate-specific antigen recurrence after radical prostatectomy: long-term follow-up, BJU Int, 2012;109:32-9.

49. Buyyounouski MK, et al., Interval to biochemical failure highly prognostic for distant metastasis and prostate cancer-specific mortality after radiotherapy, Int I Radiat Oncol Biol Phys, 2008;70:59-66

50. Antonarakis ES, et al., Changes in PSA kinetics predic metastasis-free survival in men with PSA-recurrent prostate cancer treated with nonhormonal agents: combined analysis of four phase II trials, Cancer, 2012;118:533-42.

51. Antonarakis ES, et al., The effect of PSA doubling time (PSADT) and Gleason score on the PSA at the time of initial metastasis in men with biochemical recurrence after prostatectomy, ASCO Meeting Abstracts, 2011;29(Suppl. 7):16.

52. Castellucci P, et al., Influence of trigger PSA and PSA kinetics on 11C-Choline PET/CT detection rate in patients with biochemical relapse after radical prostatectomy, I NuCl Med, 2009;50:1394-400

53. Spiotto MT, Hancock SL, King CR, Radiotherapy after prostatectomy: improved biochemical relapse-free survival with whole pelvic compared with prostate bed only for high-risk patients, Int J Radiat Oncol Biol Phys, 2007;69:54-61.

54. Stephenson AJ, et al., Predicting the outcome of salvage radiation therapy for recurrent prostate cancer after radical prostatectomy, J Clin Oncol, 2007;25:2035-41.

55. Trock BJ, et al., Prostate cancer-specific survival following salvage radiotherapy vs observation in men with biochemical recurrence after radical prostatectomy, JAMA 2008:299:2760-9

56. Boorjian SA, et al., Radiation therapy after radical prostatectomy: impact on metastasis and survival, J Urol, 2009; 182:2708-14

57. King $C R$, The timing of salvage radiotherapy after radical prostatectomy: a systematic review, Int J Radiat Oncol Biol Phys, 2012:84:104-11.

58. Bolla M, et al., Postoperative radiotherapy after radica prostatectomy for high-risk prostate cancer: long-term results of a randomised controlled trial (EORTC trial 22911), Lancet, 2012:380:2018-27.

59. Shipley WU, et al., Initial report of RTOG 9601 , a phase III trial in prostate cancer: Effect of anti-androgen therapy (AAT) 
with bicalutamide during and after radiation therapy (RT) on freedom from progression and incidence of metastatic disease in patients following radical prostatectomy (RP) with pT2-3,NO disease and elevated PSA levels, ASCO Meeting Abstracts, 2011;29(Suppl. 7):1

60. Quon $\mathrm{H}$, et al., Population-based referrals for adjuvant radiotherapy after radical prostatectomy in men with prostate cancer: impact of randomized trials, Clin Genitourin Cancer, 2014;12:e1-5

61. Hoffman KE, et al., Recommendations for post-prostatectomy radiation therapy in the United States before and after the presentation of randomized trials, J Urol, 2011;185:116-20.

62. Cipolla BG, et al., First double-blind placebo-controlled, multicenter, randomized trial of stabilized natural sulforaphane in men with rising PSA following radical prostatectomy, ASCO Meeting Abstracts, 2014;32(Suppl. 15):5032.

63. Stephenson AJ, et al., Salvage therapy for locally recurrent prostate cancer after external beam radiotherapy, Curr Treat Options Oncol, 2004;5:357-65.

64. Chade DC, et al., Salvage radical prostatectomy for radiationrecurrent prostate cancer: a multi-institutional collaboration, recurrent prostate cancer:
Eur Urol, 2011:60:205-10.

65. Rocco B, et al., Current status of salvage robot-assisted laparoscopic prostatectomy for radiorecurrent prostate cancer Curr Urol Rep, 2012;13:195-201.

66. Gotto GT, et al., Impact of prior prostate radiation on complications after radical prostatectomy, J Urol, 2010;184:136-42

67. Chade DC, et al, Cancer control and functional outcomes of salvage radical prostatectomy for radiation-recurrent prostate cancer: a systematic review of the literature, Eur Urol 2012;61:961-71.
68. Ramey SJ, Marshall DT, Re-irradiation for salvage of prostate cancer failures after primary radiotherapy, World I Urol, 2013;31:1339-45.

69. Spiess PE, et al., Outcomes of salvage prostate cryotherapy stratified by pre-treatment PSA: update from the COLD registry, World I Urol, 2013;31:1321-5.

70. Mouraviev V, Spiess PE, Jones IS, Salvage cryoablation for locally recurrent prostate cancer following primary radiotherapy, Eur Urol, 2012;61:1204-11

71. Antonarakis $\mathrm{ES}$, et al., Long-term overall survival and metastasis-free survival for men with prostate-specific antigenrecurrent prostate cancer after prostatectomy: analysis of the Center for Prostate Disease Research National Database, BJ Int, 2011;108:378-85.

72. Paller CJ, et al., The effect of PSA frequency and duration on PSA doubling time (PSADT) calculations in men with biochemically recurrent prostate cancer (BRPC) after definitive local therapy, ASCO Meeting Abstracts, 2012;30(Suppl. 15):4559.

73. Garcia-Albeniz $X$, et al., Immediate versus deferred initiation of androgen deprivation therapy in prostate cancer patients with PSA-only relapse, ASCO Meeting Abstracts, 2014;32(Suppl. with PSA
15):5003.

74. Crook JM, et al., Intermittent androgen suppression for rising PSA level after radiotherapy, N Eng/ J Med, 2012;367:895-903.

75. Figg WD, et al., A double-blind randomized crossover study of oral thalidomide versus placebo for androgen dependent prostate cancer treated with intermittent androgen ablation, J Urol, 2009;181:1104-13; discussion 1113

76. Spisek R, et al., Cancer immunotherapy of patients with the biochemical relapse of the prostate cancer using dendritic cell-based vaccine DCVAC/PCa, ASCO Meeting Abstracts, 2014,32(Suppl. 15):3099
77. Liu G, et al., Eastern Cooperative Oncology Group Phase II Trial of lapatinib in men with biochemically relapsed, androgen dependent prostate cancer, Urol Oncol, 2013;31:211-8.

78. Paller $\mathrm{CJ}$, et al., A randomized phase II study of pomegranate extract for men with rising PSA following initial therapy for localized prostate cancer, Prostate Cancer Prostatic Dis, 2013;16:50-5.

79. Pantuck AJ, et al., Phase II study of pomegranate juice for men with rising prostate-specific antigen following surgery or radiation for prostate cancer, Clin Cancer Res, 2006;12:4018-26.

80. Paller $\mathrm{CJ}$, et al.. A phase I trial of muscadine grape skin in men with biochemically recurrent prostate cancer, ASCO Meeting Abstracts, 2014:32(Suppl. 4):263.

81. Dorai T, Gehani N, Katz A, Therapeutic potential of curcumin in human prostate cancer. II. Cumin inhibits tyrosine kinase activity of epidermal growth factor receptor and depletes the protein, Mol Urol, 2000;4:1-6.

82. Thomas R, et al., A double-blind, placebo-controlled randomised trial evaluatin the effect if a polyphenol-rich whole food supplement on PSA progression in men with prostate cancer the U.K. NCRN Pomi-T study, Prostate Cancer Prostatic Dis, 2014;17:180-6.

83. Smith MR, et al., ARN-509 in men with high-risk nonmetastatic castration-resistant prostate cancer (CRPC), ASCO Meeting Abstracts, 2013;31(Suppl. 6):7.

84. MCLeod DG, et al., Phase I dose escalation trial of MVA-BN-PRO in men with nonmetastatic castration-resistant prostate cancer, ASCO Meeting Abstracts, 2013;31(Suppl. 6):193.

85. Ryan CJ, et al., Effect of abiraterone acetate and low-dose prednisone on PSA in patients with nonmetastatic castrationresistant prostate cancer: The results from IMAAGEN core study, ASCO Meeting Abstracts, 2014;32(Suppl. 15):5086. 